\title{
DIEZ AÑOS DE CONSERVACIÓN DE FLORA AMENAZADA EN CATALUÑA: ESPECIES EXTINTAS, EN PELIGRO CRÍTICO Y EN PELIGRO
}

\author{
Pascual CARbonell, David
}

\begin{abstract}
Endangered taxa are species whose conservation in the environment has been threatened. An exhaustive bibliographic survey of endangered taxa in the region of Catalonia has been conducted, for the time period (20102019). A database has been created in Excel format in order to present and review the results. Greater knowledge of the taxa enables a better classification of the risk, allowing for the adequate prioritization of conservation actions. The most important threads, as shown by the information gathered, are the invasive non-native plants and those derived from human activity. Unfortunately, due to the lack of approved conservation plans, Natural Parks have been the ones left to develop monitoring programmes (SEFA).
\end{abstract}

Keywords: Catalonia, endangered flora, critically endangered and non-native flora.

\section{Resumen}

Los taxones amenazados son especies cuya conservación en el medio natural se ha visto en peligro. Se ha realizado una búsqueda exhaustiva bibliográfica de los taxones amenazados dentro del territorio de la Cataluña autonómica durante el período (2010-2019). Para dejar constancia y exponer los resultados, se ha elaborado una base de datos en formato Microsoft Excel. El aumento del conocimiento de los taxones permite una mejor clasificación del grado de amenaza, permitiendo que la priorización de las actuaciones de conservación que se realicen sean las adecuadas. Las amenazas más importantes, según la información obtenida, son las plantas alóctonas invasoras y las derivadas de la actividad humana. Desgraciadamente, debido a la falta de planes de conservación, han sido los propios parques naturales los que han elaborado programas de seguimiento (SEFA).

Palabras clave: Cataluña, flora amenazada, en peligro, en peligro crítico y flora alóctona.

\section{Resum}

Els tàxons amenaçats són espècies dels quals la conservació en el medi natural s'ha vist en perill. S’ha dut a terme una recerca exhaustiva bibliogràfica dels tàxons amenaçats al territori de la Catalunya autonòmica durant el període 2010-2019, i per deixar constància i exposar-ne els resultats, s'ha elaborat una base de dades en format Microsoft Excel. L'augment del coneixement dels tàxons permet una millor classificació del grau d'amenaça, i permet que la priorització de les actuacions de conservació que s'implementin siguin les adequades. Les amenaces més importants, segons la informació recopilada, són les plantes allòctones invasores i les derivades de l'activitat humana. Cal assenyalar que la manca de plans de conservació de part dels estaments de govern han forçat els parcs naturals a elaborar programes de seguiment (SEFA).

Paraules clau: Catalunya, flora amenaçada, en perill, en perill crític, flora al·lòctona.

\section{Introducción}

Un ecosistema es un sistema biológico conformado por una comunidad de seres vivos y el medio físico en el que interaccionan. Se considera una unidad formada por organismos independientes que 
conviven en un mismo hábitat. Los ecosistemas suelen formar cadenas de interdependencia entre los organismos que coexisten y están unidos por el flujo de transferencia de energía y la materia orgánica (nutrientes).

Como todos los organismos, están interrelacionados en este sistema de coexistencia, por lo que la desaparición de uno de ellos puede generar grandes impactos en el ecosistema. La extinción es el proceso por el que se conoce la desaparición de todos los miembros de una especie o taxón (grupo de organismos emparentados agrupados bajo el mismo nombre). Debido a la amplia distribución biogeográfica que pueden tener las especies, determinar cuándo una está extinta puede ser muy complicado. Según los criterios internacionalmente reconocidos de la International Union for Conservation of Nature (UICN), se considera extinto un taxón si no ha habido contactos fidedignos en los últimos cincuenta años (DOGC, 2015), mediante la realización de prospecciones exhaustivas de sus hábitats, conocidos o esperados, en los momentos apropiados y a lo largo de su área de distribución histórica (UICN, 2012).

Debido al impacto ocasionado por la extinción de una especie, se requiere llevar un seguimiento de todas las especies que estén bajo algún grado de amenaza. En 1948 se creó la UICN, que se ha convertido en la autoridad mundial en cuanto a evaluar el estado de conservación de la naturaleza y los recursos naturales, así como de promover las medidas necesarias para protegerlos. Los conocimientos y las herramientas que la UICN suministra son cruciales para posibilitar el progreso humano, el desarrollo económico y la conservación de la naturaleza (IUCN, 2020). Una de estas herramientas son las «listas rojas», en las que se clasifican las especies que están amenazadas. Se pretende que las categorías y criterios de la Lista Roja de la UICN constituyan un sistema de fácil comprensión para clasificar especies de alto riesgo de extinción global. El fin general es proporcionar un marco explícito y objetivo para la clasificación del espectro más amplio posible de especies según su riesgo de extinción (UICN, 2012).

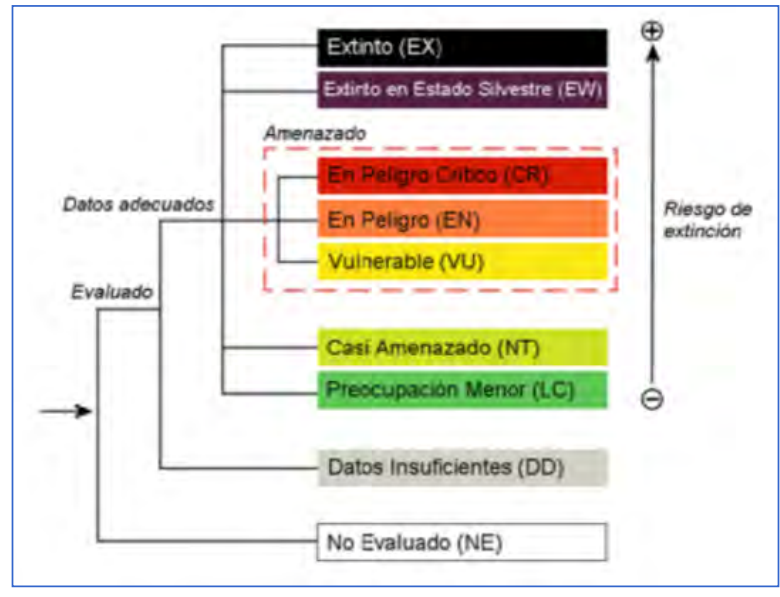

Figura 1. Estructura de las categorías (UICN, 2012).

La estructura de las categorías en las que se distribuyen las especies incorporadas en la Lista Roja es la que se muestra en la figura 1 . Se diferencian en función de la cantidad y fiabilidad de los datos, además de su clasificación en función del riesgo de extinción.

Debido al vacío que existía en cuanto a la ordenación y la síntesis de la flora vascular de especial interés conservacionista se redactó la Ley 42/2007 del patrimonio natural y de la biodiversidad, que establecía que las comunidades autónomas pudieran establecer catálogos de especies amenazadas, y determinar así las prohibiciones y actuaciones suplementarias que se considerarán necesarias para su conservación (DOGC, 2015). Esta ley viene a derogar y sustituir la Ley 4/1989 de conservación de los espacios naturales y de la flora y fauna silvestres que, a su vez, procedía de la Ley de 1975, de espacios naturales protegidos (BOE, 2007). Esta ley es la que se mantiene en vigor, y algunos artículos fueron modificados por la Ley 33/2015 (BOE, 2015a). 
Más tarde, entró en vigor el Decreto 172/2008 mediante el que se creó el Catàleg de flora amenaçada de Catalunya (CFA) (DOGC, 2008), a partir del cual se aprobaron diversas normas que incidían sobre su contenido, siendo una de estas el Real Decreto 139/2011 para el desarrollo de la Lista de especies silvestres en régimen de protección especial (LESPE) y el Catálogo español de especies amenazadas (CEEA) (BOE, 2011). En el CFA se establece un listado de taxones amenazados en el territorio catalán y se clasifican según dos criterios legales: vulnerable o en peligro de extinción.

Finalmente, se publicó en el Diari Oficial de la Generalitat de Catalunya (DOGC) la resolución AAM/732/2015 por la que se aprueba la ampliación de especies del CFA (DOGC, 2015).

Además, en Cataluña, para la síntesis de la flora vascular de especial interés conservacionista, se comenzó en 2003 la elaboración de una obra que sería publicada en 2010 bajo el título de Llibre Vermell de les plantes vasculars endèmiques i amenaçades de Catalunya, referido, en este trabajo, de ahora en adelante como Libro Rojo. Gracias a esta obra, se dispone de un listado de todas las plantas vasculares amenazadas y endémicas de Cataluña clasificadas según los criterios de la UICN, junto con una monografía de cada especie.

El proyecto del Libro Rojo fue presentado a los organismos pertinentes para concienciar y facilitar la gestión de la conservación de las especies y taxones listados en su interior. Además, los catálogos de especies amenazadas comentan la obligación de elaborar y aprobar planes de recuperación para las especies y subespecies en peligro de extinción y planes de conservación para las vulnerables, herramientas que han de garantizar la preservación y la mejora de las poblaciones de los taxones. No obstante, la redacción y ejecución de estos planes no se ha desarrollado en Cataluña (Blanché, 2013).

Con el objetivo de disponer de un plan que guiara la actuación de la Generalitat de Cataluña en el ámbito de la flora amenazada, en 2014 se encargó a la ICHN un documento que sirviera de base para la aprobación de la Estrategia catalana de conservación de la flora, que aún está pendiente de aprobación por parte del Gobierno de la Generalitat de Cataluña (Generalitat de Catalunya, 2020a).

La situación actual, con ningún plan de conservación o de recuperación aprobado, hace necesario conseguir algún tipo de herramienta que permita salvaguardar de manera urgente las poblaciones con un riesgo importante. Por este motivo, algunas instituciones han optado por elaborar unos documentos provisionales que son los programas de conservación. Estos documentos plantean actuaciones clave factibles, a la espera de que se puedan elaborar y desarrollar los planes que marca la normativa vigente (Oliver, 2018).

\section{Metodología}

Para la realización de este trabajo, se ha elaborado una base de datos en formato Microsoft Excel de los taxones amenazados. Este Excel, un fragmento del cual se puede observar en la figura 2, está estructurado de tal forma que cada línea es un taxon.

Está conformado por la suma entre los taxones obtenidos del Libro Rojo (Sáez et al., 2010) junto con la resolución de la ampliación del CFA (DOGC, 2015) y las nuevas incorporaciones a la flora catalana que se han catalogado en el período de tiempo de estudio. El resultado consta de un listado de 354 taxones.

Por otro lado, las columnas son los distintos ámbitos de información encontrada. Se considerará, exclusivamente, la información de los últimos diez años (2010-2019) siempre que sea nueva en relación con lo publicado en el Libro Rojo (Sáez et al., 2010). Las columnas son las siguientes:

- Ampliación del conocimiento:

- Datos bibliográficos de los taxones:

- Nombre científico.

- Número de orden: código interno asignado.

- Familia botánica.

- Sinónimos. 


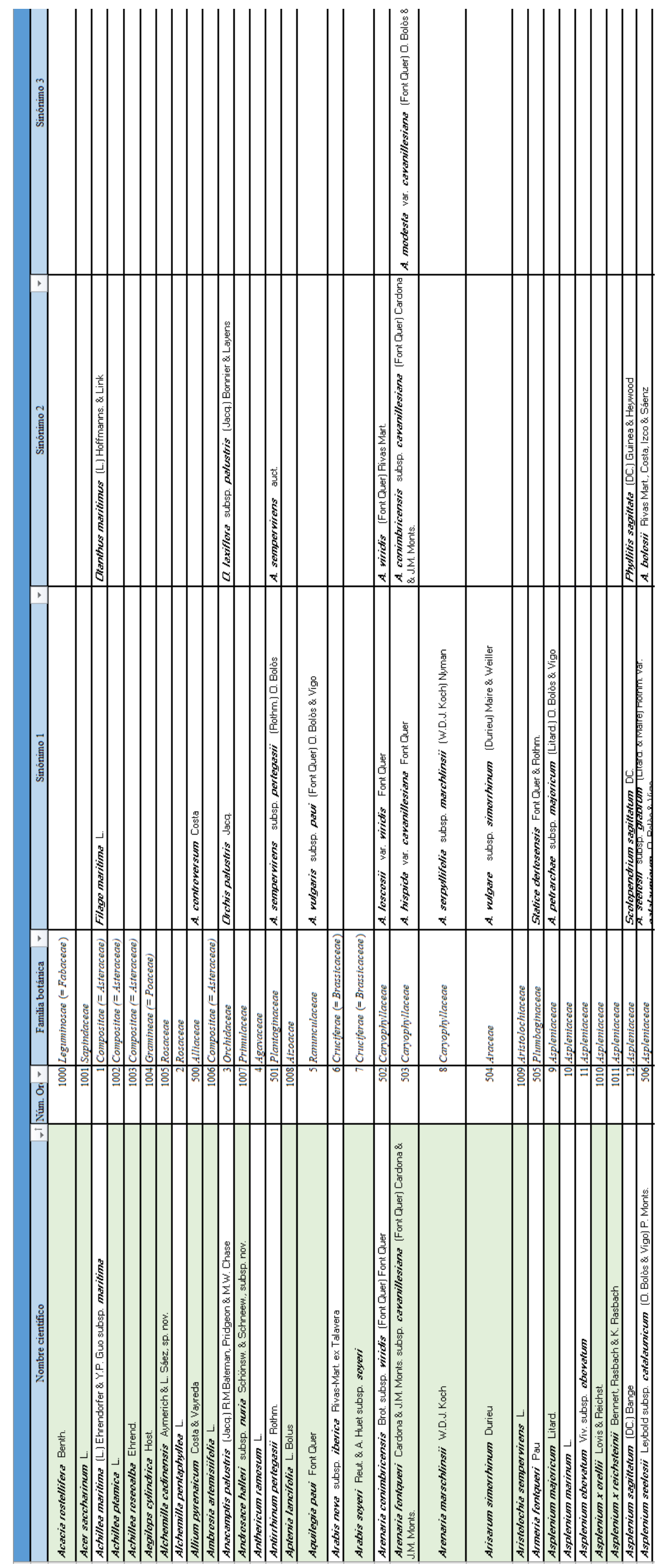

David Pascual CaRbonell

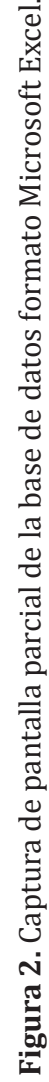


- Categoría UICN.

- Endémico o no endémico.

- Taxón añadido: marcados con X los taxones añadidos como nuevos en la resolución AAM/732/2015 (DOGC, 2015).

- Taxón eliminado: marcados con X los taxones descatalogados en la resolución AAM/732/2015 (DOGC, 2015).

- Taxón cambiado de categoría: marcados con X los taxones especificados como cambio de categoría legal en la resolución AAM/732/2015 (DOGC, 2015).

- Discrepancias: se evidencian las discrepancias de categoría entre la asignación de nivel legal frente al criterio UICN.

- Corología:

- Localidad.

- Hábitat.

- Demografía:

- Censo.

- Edad de la población.

- Proyección demográfica.

- Viabilidad poblacional.

- Ecología:

- Agrupaciones.

- Relaciones de mutualismo.

- Amenazas bióticas:

- Competencia interespecies.

- Plagas.

- Ganado.

- Depredación.

- Amenazas abióticas:

- Construcción.

- Contaminación.

- Cambio climático.

- Destrucción del hábitat.

- Alteración del hábitat.

- Genética:

- Cariograma.

- Número cromosómico.

- Cantidad de ADN.

- Tamaño del genoma.

- Filogenia.

- Evolución.

- Marcadores: nucleares, cloroplásticos y enzimáticos.

- Gestión:

- Incorporación a nuevas listas de especies protegidas estatales:

- LESPE:

- Se asignó X a aquellos taxones incorporados en la LESPE; CEEA 2011 a aquellos taxones incorporados en el CEEA (BOE, 2011), y CEEA 2015 a aquellos incorporados en la Orden AAA/1771/2015 (BOE, 2015b).

- Ampliación del catálogo.

- Otras regulaciones.

- Evaluación de criterios.

- Planes redactados:

- Planes de gestión de parques naturales. 
- Planes de recuperación redactados.

- Medidas de gestión del hábitat en áreas protegidas o no:

- Regulación de acceso.

- Restauración del hábitat.

- Medidas de gestión poblacionales:

- Refuerzo.

- Reintroducción.

- Translocación.

- Otros proyectos.

- Medidas de seguimiento:

- Administración.

- Entidades.

- Particulares.

- Programas de formación: estudiantes, escolares, profesionales y público general.

- Conservación ex situ:

- Banco de germoplasma.

- Banco de polen.

- Multiplicación in vitro.

- Jardines botánicos.

- Herbarios.

- Otros centros.

- Difusión del conocimiento: rotulación, cartelería, paneles, señalización, publicación de monografías, pósteres, exposiciones o conferencias, periódicos, portales online y centros.

Se ha realizado una exhaustiva búsqueda de información del período de estudio (2010-2019) en las siguientes fuentes bibliográficas:

- BioC (BioC, 2020)

- Butlletí de la Institució Catalana d'Història Natural

- Collectanea Botanica

- Orsis

- Acta Botanica Barcinonensia

- Willdenowia

- Annals de la Delegació de la Garrotxa de la ICHN

- Anales del Jardín Botánico de Madrid

- Conservation Biology

- Biological Conservation

- Oryx

- Global Ecology and Conservation (GECCO)

- Biodiversity and Conservation

- Grup de Naturalistes d'Osona (GNO, 2020a)

- Portal online del Centro de Investigación Ecológica y Aplicaciones Forestales (CREAF, 2020)

- Portal online de Parcs de Catalunya Xarxa de Parcs Naturals:

- Trobada d'Estudiosos del Montseny

Trobada d'Estudiosos de les Garrigues

Trobada d'Estudiosos de Sant Llorenç del Munt i l'Obac

Trobada d'Estudiosos de la Serralada Litoral Central

Trobada d'Estudiosos del Montnegre i el Corredor

Trobada d'Estudiosos del Garraf i d'Olèrdola

Trobada d'Estudiosos del Foix

- Portal online de la librería de la Diputación de Barcelona: 
- Pla de conservació del Parc Natural del Montseny. Reserva de la Biosfera

- Pla de conservació del patrimoni natural i de la biodiversitat dels parcs del Garraf i d'Olèrdola

- Se revisaron las siguientes actas de congresos:

- Jornades de Conservació de Flora i Funga (III y IV)

- Congreso de Biología de la Conservación de Plantas $\left(5 .^{\circ}, 7 .^{\circ}, 8 .^{\circ}\right.$ y 9.)

\section{Resultados y discusión}

Los resultados de la búsqueda bibliográfica se presentan agrupados en una base de datos en formato Microsoft Excel que, por su tamaño (356 filas x 81 columnas $=28.836$ celdas), no se reproduce en la memoria y se halla disponible en el Campus Virtual de la Universidad de Barcelona en la dirección: $<$ https://campusvirtual.ub.edu/mod/resource/view.php?id=1411077>.

\subsection{Mejora en el conocimiento de los taxones}

\subsubsection{Análisis de los datos bibliográficos de los taxones}

La base de datos se ha creado a raíz de un listado de 199 taxones extraídos del Libro Rojo (Sáez et al., 2010) pertenecientes a la categoría de vulnerable (VU) o superior, siendo estas las categorías de «en peligro» (EN), «en peligro crítico» (CR), «regionalmente extinto» (RE) y «extinto» (EX). Seguidamente, se incorporaron 66 taxones incluidos en el CFA y en la ampliación mediante la resolución AAM/732/ 2015 (DOGC, 2015). Finalmente, se hallaron 89 taxones considerados como nuevos para la flora catalana. Como resultado se obtuvo un listado de 354 taxones de los cuales un 56,22\% corresponden al Libro Rojo, un $18,64 \%$ al CFA y un $25,14 \%$ a flora nueva para Cataluña. Cabe remarcar que estos porcentajes hacen referencia a la fuente de origen de la inclusión del taxón en el listado, es decir, muchos taxones pertenecientes al CFA no fueron añadidos como tales, porque ya se habían incorporado a raíz del Libro Rojo. Los taxones del listado se reparten entre 96 familias botánicas. Se ha recopilado información de un total de 201 taxones que representan el 56,78\% del total.

En la figura 3 se puede observar la distribución de los taxones entre las distintas categorías de amenaza según los criterios de la UICN.

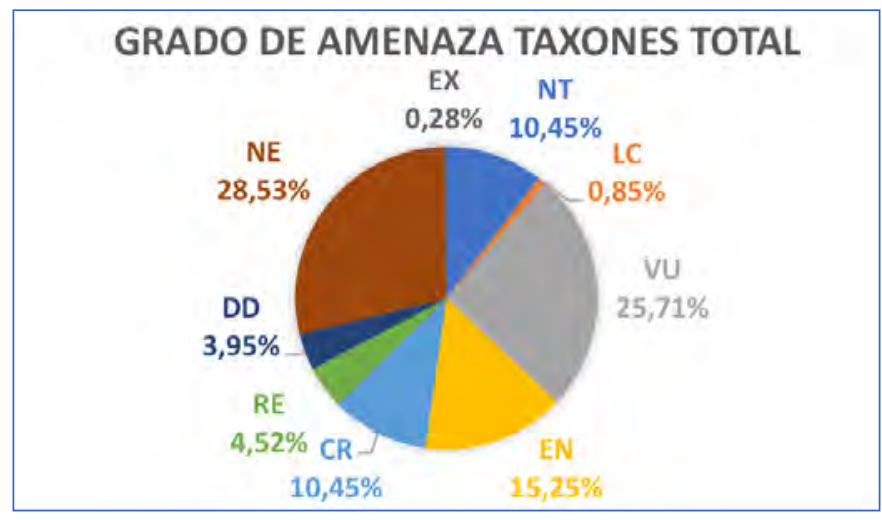

Figura 3. Distribución de los taxones según el grado de amenaza.

Es curioso observar cómo las categorías de «preocupación menor» (LC), «casi amenazado» (NT), «datos insuficientes» (DD) y «no evaluado» (NE) que no estaban consideradas en la elaboración del listado inicial, aquí representan el $43,78 \%$ del total de los taxones. La categoría con más taxones per- 
tenece a los NE. Esta categoría puede ser confusa al hacernos creer que los taxones incluidos en ella no están amenazados, pero simplemente son taxones que no han sido evaluados y esto puede ser debido tanto al desconocimiento previo de su existencia y, por tanto, no ha habido tiempo de hacer prospecciones del grado de amenaza, como aquellos que realmente no se pueden considerar como amenazados y por ello no han sido catalogados. En segundo lugar, encontramos los taxones VU, que son para los que se deberían realizar planes de conservación. Seguidamente, los taxones EN, CR y NT, con más de un 10\% del total, son categorías (excluyendo los NT) en las que se deberían realizar planes de recuperación (Generalitat de Catalunya, 2020a).

Si dividimos los taxones en función del endemismo catalán o no, la mayoría de ellos no son endémicos. Representan el $86,16 \%$ del total. Curiosamente, se suele pensar que las especies endémicas son más vulnerables por el hecho de presentar un área de distribución más limitada y ser números poblacionales más reducidos. Pero estos resultados indican que esta concepción no es cierta. Las especies más amenazadas son no endémicas, esto puede ser debido a que Cataluña represente el límite de su área de distribución y que por ello estén más empobrecidas genéticamente, o aisladas, o que no presenten la posibilidad de ser rescatadas, tanto poblacional como genéticamente, por el grueso de la población o porque en nuestro territorio padezcan una presión más intensa.

En lo referente a los cambios realizados en el CFA, se añadieron 67 nuevos taxones que se categorizaron como: 1 NT, 32 VU, 17 EN, 9 CR, 2 RE, 1 EX, 3 DD y 2 NE. Aparte, se descatalogaron cinco taxones (VU) que son Carex grioletii, Euphorbia dendroides, Erodium rupestre [= Erodium foetidum subsp. rupestre], Salsola soda y Saxifraga longifolia subsp. aitanica [= Saxifraga longifolia subsp. longifolia var. aitanica]. Además, hubo trece cambios de categoría legal, donde nueve fueron cambios de VU a EN, lo que supone un aumento de nivel legal de protección, y cuatro mejoraron su situación (cambio de EN a VU) siendo estos taxones: Kosteletzkya pentacarpa, Reseda jacquinii subsp. litigiosa, Salix foetida y Zygophyllum album.

Por último, la categoría legal del CFA y la de la UICN no tienen por qué coincidir. Pese a que la categoría de la UICN es más precisa, la que prevalece a la hora de priorizar planes de conservación y recuperación es la categoría legal. Se evidenciaron veintisiete discrepancias entre la clasificación legal y la de la UICN. Estaban clasificados como VU veintidós taxones que, según la UICN, presentan un grado de amenaza mayor. Por otro lado, encontramos cinco taxones que están más protegidos de lo necesario, pertenecientes a la categoría EN legal, mientras que, según criterios de la UICN, les correspondería un grado de amenaza inferior.

\subsubsection{Corología, demografía y ecología}

Los datos corológicos han sido los más abundantes, junto con el censo y los depósitos herbarios que se verán más adelante.

Este elevado número de referencias encontradas es debido, principalmente, a la tipología mayoritaria de las publicaciones. Se trata de catálogos florísticos que son documentos que exponen un listado de los taxones localizados en un área determinada. La información que se transmite es la referente al lugar donde se ha encontrado el taxón, el hábitat, señalización del cuadrante UTM, censo aproximado de los individuos encontrados, el margen altitudinal y el código del depósito del testimonio en el herbario, así como una breve explicación de la situación del taxón.

La entrada más abundante ha sido la de localidades. En esta entrada hay un recopilatorio de todas las zonas en las que se encontró un taxón determinado. Estos lugares se dividen en cuatro tipologías: aquellos en los que ya se conocía la presencia del taxón y ha sido confirmada como aún presente; aquellos que suponen una novedad; aquellos en los que se conocía la presencia del taxón, pero no se ha podido confirmar, y aquellos en los que se consideraba desaparecido el taxón y se ha vuelto a reencontrar. Los de notable mención son el caso de Gentiana pneumonanthe, un taxón CR cuya extinción local se confirmó en Santa Caterina y su desaparición en Ulldeter (Aymerich et al., 2010) y el caso de ocho taxones que fueron redescubiertos en localidades donde se creían extintos. Estos son Carex 
brevicollis (VU) (Soriano y Aymerich, 2017), Delphinium bolosii (EN) (BioC, 2012d), Delphinium montanum (VU) (Aymerich, 2015), Hydrocotyle vulgaris (EN) (Jover Benjumea et al., 2014), Isoetes durieui (NT) (Guardiola y Gutiérrez, 2012), Potamogeton perfoliatus (EN) (Aymerich, 2012), Reseda hookeri (RE) (Guardiola y Romera, 2018) y Spirodela polyrrhiza (RE) (Aparicio et al., 2013; Curto et al., 2013).

En cuanto a los hábitats, la mayoría ya eran conocidos para las especies tratadas y solo un taxón (Arabis soyeri subsp. soyeri) fue encontrado en un entorno atípico (Aymerich, 2013a).

El bloque de demografía consta del censo, la edad poblacional, proyección demográfica y viabilidad poblacional. Estos cuatro ítems poseen un valor muy elevado a la hora de determinar el grado de amenaza, ya que en su categorización se tiene en cuenta el estado actual del taxón y su posible estado en un futuro. Para esta determinación se considera el número de individuos, su grado de madurez para determinar su viabilidad mediante reproducción sexual o vegetativa y la distribución de las poblaciones, ya que son factores de vital importancia para la dispersión y crecimiento poblacional (UICN, 2012).

Finalmente, están las consideraciones ecológicas en las que se engloban las agrupaciones y las relaciones de mutualismo. Las agrupaciones hacen referencia a las especies, comunidades y alianzas con las que conviven los taxones estudiados.

Las relaciones de mutualismo son importantes para conocer más acerca de la viabilidad poblacional mediante reproducción sexual, ya que esta categoría cataloga las vías de dispersión y polinización. Esto se refleja en el curioso caso de Aquilegia paui, una especie que pese a consumir recursos en la producción de flores para atraer polinizadores, debido a una reducción de las visitas de estos se ha empezado a observar un aumento en la reproducción por autogamia (Martinell et al., 2011b).

Con el fin de realizar unos planes de conservación y protección adecuados, son necesarios programas de seguimiento periódicos. Existen factores que, si los tenemos en consideración, nos pueden beneficiar a la hora de hacer estas determinaciones. Uno de estos factores son las agrupaciones, dado que permiten determinar lugares que, tanto por la disposición geográfica como por las especies que crecen allí, pudieran ser hábitats potenciales para taxones amenazados.

\subsubsection{Amenazas}

Otros factores determinantes a la hora de clasificar el grado de riesgo de las especies son las amenazas a las que se ven expuestas. Estas pueden ser bióticas, si son debidas a otros organismos, o abióticas, si no son debidas a otros organismos.

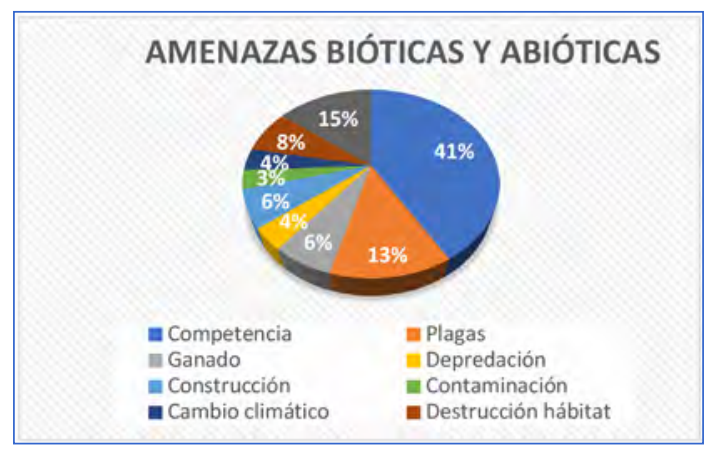

Figura 4. Distribución de las amenazas.

En la figura 4 vemos la proporción de las amenazas sobre el total de información recopilada. Es destacable el gran porcentaje que ocupa la competencia entre especies. Esta competencia es tanto por el espacio físico como por los nutrientes o la luz solar. Aún más remarcable es el hecho de que el $79,22 \%$ de las entradas de competencia son de plantas alóctonas, es decir, especies que han sido introducidas. El peligro que supone la introducción de estas plantas depende de su potencial inva- 
sor y su capacidad para alterar el ecosistema, que está relacionada con una alta capacidad reproductiva y adaptación a cualquier medio. Las especies alóctonas pueden causar cambios en los ecosistemas donde se introducen, llamados impactos, que pueden ser potencialmente dañinos para la biodiversidad (Jeschke et al., 2014). Mantener un listado de especies alóctonas e invasoras permite tener un seguimiento de estos taxones para valorar el peligro que suponen e idear actuaciones en consecuencia.

Esto se correlaciona con otro de los tres grandes grupos de amenazas registrado: las plagas. En esta categoría, un $91,67 \%$ de la información se refería al potencial invasor de los taxones tratados (36,06\% de las entradas de plantas alóctonas). Solo Spirodela polyrrhiza (RE) era considerado con comportamiento invasor, sin ser una planta alóctona, ya que desplazaba del hábitat a Lemna minor (Conesa et al., 2016).

Las otras dos entradas de plagas son los casos de Delphinium bolosii (EN), que se ha visto afectado por el crecimiento de un hongo (Synchytrium aureum) que reseca las hojas (Bosch et al., 2019a) y Salix lapponum (NT), asolado por una enfermedad extraña que mataba las partes aéreas (Aymerich, 2017).

El último gran bloque es el que pertenece a la categoría de «alteración del hábitat». Si se modifican las condiciones, el terreno o el ecosistema en el que viven las especies, es probable que les repercuta negativamente e incluso que se vuelva inviable su presencia. De las veintisiete entradas obtenidas, veintiuna $(77,78 \%)$ hacen referencia a la actividad humana y su impacto. Además, las categorías de construcción (6\%), contaminación (3\%) e, indirectamente, el cambio climático (4\%) también son debidos a la actividad humana. Es decir, el 28\% del total de información sobre amenazas deriva de actividades humanas.

Uno de estos peligros que no se ha visto muy reflejado en los resultados pero que, sin duda, supondrá un peligro en el futuro es el cambio climático. No son pocos los estudios que están comenzando a surgir respecto a esta amenaza, valorando el impacto que tendrá sobre especies vulnerables. En nuestra búsqueda han sido pocas las referencias que se han hecho hacia este factor, pero las pocas que han surgido hacían constar la evidente preocupación de la alteración en los hábitats que puede generar el cambio climático, principalmente por el incremento de temperatura y la disminución de las precipitaciones, afectando en particular a especies de hábitats alpinos.

En ningún caso, las amenazas aquí recopiladas sustituyen a las incluidas en el Libro Rojo, sino que son factores sumatorios a los ya conocidos.

\subsubsection{Genética}

Otro tipo de publicaciones encontradas han sido los estudios genéticos. Estos artículos se centraban en el análisis del parentesco y caracterización de especies, así como en el reconocimiento de alelos exclusivos para determinar la variabilidad genética. Esta determinación sirve para valorar la prioridad e importancia de la conservación de poblaciones determinadas de un taxón, es decir, si un taxón amenazado tuviera una población aislada en gran riesgo, si esta fuera la más pobre genéticamente en comparación con las otras poblaciones, no supondría una gran pérdida para la especie. Para la determinación de estos alelos se realizan varios ensayos que usan distintos marcadores nucleares, cloroplásticos y enzimáticos.

El apartado que más entradas recogió fue filogenia. En este apartado se evidencia la ascendencia de los taxones, así como la confusión con otras especies. Distinguir dos especies similares morfológicamente es muy complejo. De esto queda constancia en el hecho de que el $47,77 \%$ de las entradas de filogenia hacen referencia a este tipo de confusiones, esto es, que un taxón había sido confundido por otro. La mayoría de los taxones confundidos son especies NE, pero también encontramos especies como Aquilegia paui (EN) y Potamogeton lucens (CR), que están altamente amenazadas.

Se ha visto demostrada la importancia de la identificación de especies mediante técnicas genéticas. La importancia de estos datos recae en el hecho de que los censos y valoraciones de las poblaciones son factores cruciales en la determinación de los grados de amenaza y, si no se identifican co- 
rrectamente, se estará incurriendo en el error de sobrevalorar una especie o infravalorarla y, como consecuencia, determinar que requiere una protección mayor o menor de la necesaria.

\subsection{Mejora en la gestión de los taxones y difusión del conocimiento}

\subsubsection{Incorporación a listas estatales y evaluación de criterios de la UICN}

La distribución biogeográfica de una especie puede alterar y diluir el nivel de amenaza en función del nivel territorial que estemos evaluando. Una especie muy amenazada en Cataluña puede no serlo en ámbito estatal porque presente abundantes poblaciones en otras comunidades autónomas. Este hecho queda reflejado en que solo existen treinta y cinco entradas $(<10 \%$ del total) de especies protegidas por la LESPE o el CEEA, que son las listas de protección estatal de especies amenazadas. Reforzando la idea presentada anteriormente, dieciséis de estas entradas (45,71\%) representan especies endémicas del territorio catalán, es decir, que no están presentes en ningún otro lugar fuera del ámbito autonómico de Cataluña.

Por otro lado, en la columna de «otras regulaciones» se evidencia la presencia de los taxones en otras listas de protección, ya sea de otros estados - Trisetum spicatum subsp. ovatipaniculatum en la Lista Roja andorrana (Guardiola et al., 2013b) -, otras comunidades autónomas -Asplenium majoricum en el catálogo valenciano de especies amenazadas (Ibars y Estrelles, 2011) - o a nivel de localidades dentro del territorio catalán -Arisarum simorrhinum en la Lista Roja provisional del PN del Garraf (Gutiérrez y Guardiola, 2012).

Aparte de listas de protección, también se mencionan listados de especies alóctonas e invasoras. Los taxones Tradescantia pallida y T. zebrina ya han sido asignados como especies alóctonas establecidas en ámbito nacional (Aymerich, 2016a). Luego tenemos el caso de Vitis riparia, una reciente colonización que podría suponer un peligro, ya que esta especie está incluida en listados oficiales de especies invasoras de otros países (Aymerich, 2013b). Y, finalmente, la necesidad de seguimiento y de realizar las actuaciones pertinentes queda reflejado en el caso de Ambrosia artemisiifolia. Esta especie no solo puede perjudicar el ecosistema que invade, sino que está considerada como un problema para la salud pública por el inmenso potencial alergógeno del polen que produce. Este polen puede viajar hasta $100 \mathrm{~km}$ y con bajas densidades provoca alergias (entre 6-10 granos de polen $/ \mathrm{m}^{3}$ de aire en personas sensibles y a partir de 10 en personas no sensibles). Además, sus frutos pueden causar enfermedades en el ganado que los ingiere y la propia especie se considera una plaga como mala hierba de cultivos de girasol, remolacha azucarera, maíz y cereales, provocando pérdidas económicas (Oliver et al., 2010; 2016). Pese a que medidas de gestión serían necesarias, la propia biodiversidad ha acudido a ayudar en este problema, ya que en 2013 apareció un coleóptero competidor (Ophraella communa) que ha demostrado reducir el polen de esta especie en un 82\% (Rincon, 2020).

Por último, la columna de evaluación de criterios recoge todas aquellas menciones en las que se debería reconsiderar el grado de amenaza establecido. Empezamos mencionando que el 30,43\% de esta columna representa taxones NE. Este hecho pone en evidencia lo que se exponía al principio, que la categoría NE podía generar confusión al creer que los taxones pertenecientes a ella no estaban amenazados. Como resultado de las evaluaciones, encontramos clasificadas estas especies NE entre niveles de NT a CR. Esta última, la más amenazada, corresponde a Limonium ilergabonum una especie nueva recientemente descubierta (López-Alvarado et al., 2017). De entre los taxones que ya estaban clasificados, de los 16 que han sido reevaluados, la mayoría (56,25\%) se mantienen en el mismo grado de amenaza. Solo cuatro rebajan la categoría del grado de amenaza, de entre los que encontramos el caso de Reseda hookeri, que pasa de RE a CR (Guardiola y Romera, 2018). Otro caso remarcable es el de Woodsia alpina (VU), una especie cuyo nivel de amenaza consideran necesario rebajar, mientras que otros creen oportuno mantenerlo (Aymerich et al., 2010; Guardiola et al., 2013b; Oliver Martínez-Fornés, 2012b). Como último caso destacable tenemos el de Seseli farrenyi, única especie a la que se le ha aumentado el grado de amenaza de EN a CR (López-Pujol et al., 2010). 


\subsubsection{Planes redactados}

La situación actual, con ningún plan de conservación o de recuperación aprobado, ha obligado a elaborar unos documentos provisionales, los programas de conservación, que sirven como herramienta para hacer frente a la situación extrema de algunas localidades de taxones amenazadas (Oliver, 2018).

Uno de estos programas es el Programa estandarizado de seguimiento de flora amenazada de la Red de Parques Naturales de la Diputación de Barcelona (SEFA). Este programa deriva del marco de la estrategia de conservación de la flora vascular amenazada de la Red de Parques Naturales de la Diputación de Barcelona y tienen como objetivos facilitar la vigilancia sobre el territorio, incrementar el conocimiento de los taxones en seguimiento y evaluar las tendencias para elaborar adecuadamente los planes de gestión (Carrera et al., 2016). El 71,435\% de las entradas de «planes redactados» pertenecen al Programa SEFA. Los parques naturales mencionados durante la búsqueda de información han sido: PN del Garraf y Olèrdola, PN de la Serralada de la Marina, Serralada Litoral y del Montnegre y el Corredor, PN del Montseny y PN de Sant Llorenç del Munt y Obac.

Encontramos un caso curioso de confrontación de dos planes de conservación en el PN del Ports. Este es el caso de la conservación de Aquilegia paui con la conservación de Capra pyrenaica subsp. hispanica, ya que este caprino depreda al taxón amenazado (Martinell et al., 2011a).

Otra herramienta útil son los planes de conservación del espacio de interés, pese a que solo dos han sido redactados: PN del Montseny y PN del Garraf y Olèrdola. Estos planes son una herramienta de gestión básica para asegurar la conservación del patrimonio natural salvaguardándolo y protegiéndolo (Anton et al., 2014). Un 42,86\% de las entradas en la redacción de planes de parques naturales corresponde a especies dentro del marco de actuación de estos planes de conservación.

Finalmente, al no haberse elaborado ningún plan de recuperación como tal, en el apartado de planes de recuperación hemos introducido aquellas medidas y estrategias de conservación específicas para diferenciarlas de las realizadas por parques naturales. De esta forma, encontramos medidas de conservación específicas de especies, como por ejemplo Delphinium montanum, así como cinco especies dentro del marco de la estrategia catalana de conservación ex situ de la flora vascular silvestre de Cataluña (Generalitat de Catalunya, 2020b).

\subsubsection{Medidas de gestión}

Las medidas de gestión las podemos dividir en tres grandes bloques: las de gestión del hábitat (regulación de acceso y restauración), las de gestión poblacional (refuerzo, reintroducción y translocación, entre otras) y las de seguimiento (administración, entidades y particulares).

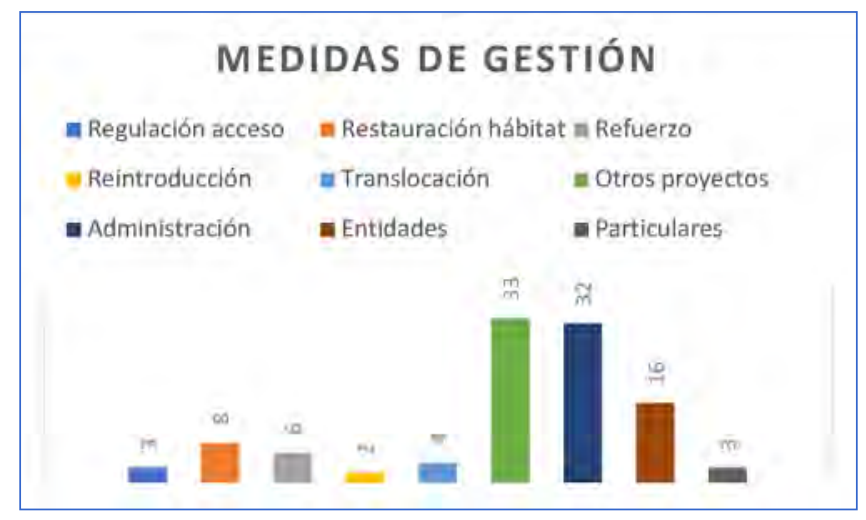

Figura 5. Distribución de los registros por medida de gestión. 
En la figura 5 queda constancia de que no se han encontrado muchas menciones sobre este tipo de proyectos. La columna con más entradas ha sido la de «Otros proyectos», donde se engloban distintas actuaciones, aunque la mayoritaria, con un $60,61 \%$ de las entradas, son ensayos de germinación. Este tipo de ensayos forman parte de la conservación ex situ, mientras que el resto son medidas in situ. Pese a que la conservación in situ debería ser la más fomentada, observamos una gran tendencia hacia los proyectos ex situ.

Las otras dos columnas con abundancia de entradas son las englobadas en las medidas de seguimiento. En este bloque se exponen las entidades privadas y las de la Administración pública que se encargan de llevar a cabo estas medidas de seguimiento, así como de financiar las operaciones. La Administración pública es quien más veces ha sido mencionada a lo largo de la búsqueda, con un total de treinta y dos entradas, de las cuales un 59,38\% pertenecen a la Red de Parques Naturales de la Diputación de Barcelona. Esta red está formada por doce espacios naturales con el objetivo de planificarlos y gestionarlos mediante planes especiales, con tal de proteger los valores naturales (Diputació de Barcelona, 2020). Y, respecto a las entidades, de las dieciséis entradas, un 68,75\% pertenecen a delegaciones de la ICHN.

\subsubsection{Conservación ex situ}

Las actuaciones para la conservación de especies y poblaciones que se desarrollan fuera del ambiente natural constituyen la llamada conservación ex situ. Se consideran actuaciones complementarias a la conservación in situ, que es la que siempre hay que fomentar de manera prioritaria. Pero, cuando esta ya no es posible, o su efectividad es limitada, puede resultar más adecuado el desarrollo de actuaciones ex situ. Los jardines botánicos son instituciones de gran interés para apoyar este tipo de actuaciones (Ministerio para la Transición Ecológica y el Reto Demográfico, 2020b).

Los jardines botánicos, además de ser centros en los que se cultiva y conservan especies, también presentan un área destinada a la conservación de la diversidad genética, mediante la colección de material vegetal vivo, en forma de semillas, esporas, polen o fragmentos vegetales; estas áreas son los llamados bancos de germoplasma. Los más mencionados durante la búsqueda de información han sido el Jardín Botánico de Barcelona, el Jardín Botánico Marimutra y el Arborétum-Jardín Botánico de Lérida (AJBLL).

Finalmente, la entrada más abundante de esta categoría, que representa el 24,01\% del total, ha sido la de los herbarios. Estos centros recopilan plantas o partes de plantas desecadas para ser identificadas. Esta identificación se realiza mediante una etiqueta en la que aparece toda la información que permite su localización y reconocimiento. Estos herbarios son elaborados por distintas entidades como institutos o universidades. Los más mencionados dentro del área autonómica de Cataluña son el Herbario del Instituto de Barcelona (BC), el Herbario de la Universidad de Barcelona (BCN), el Herbario de la Universidad de Gerona (HGI) y el Herbario del Instituto de Estudios Ilerden$\cos ($ HBIL).

\subsubsection{Difusión del conocimiento}

La difusión del conocimiento es esencial para garantizar una correcta gestión y actuación sobre cada especie. Al tener cada especie sus peculiaridades, cuanto más se sepa al respecto más sencillo será predecir los comportamientos y hacer proyecciones futuras. Un elevado nivel de conocimiento acerca de las especies permite asegurar la máxima precisión en el momento de hacer las evaluaciones y asignaciones de grados de amenaza, y evitar incurrir en errores que puedan desencadenar una sobreprotección o infravaloración de taxones.

La mayor parte de la información recopilada ha sido mediante artículos publicados en diversas revistas y libros, el 89,34\% de los taxones tiene una entrada en «publicaciones de monografías». 
Los dos siguientes grupos con más entradas, aunque representan menos de un $20 \%$ en comparación con las publicaciones, son los pósteres y conferencias. Ambos medios de difusión comparten un mismo escenario que es el de los congresos. En estos eventos suele haber comunicaciones tanto orales como gráficas (mediante pósteres), cuya información se recopila en libros de resúmenes. Hay que remarcar que mucha de la información recopilada en publicaciones se expone más tarde en este tipo de eventos.

Los portales online de diversos centros de investigación, así como de la Administración pública, han resultado una buena fuente de información.

En general, se aprecian diversas formas de difusión del conocimiento científico; la primordial consiste en la publicación de artículos en revistas de divulgación científica. Pese a que sería interesante fomentar la difusión al público general (para la concienciación social del impacto humano sobre el medioambiente), son los expertos los que tienen más peso en la toma de decisiones referentes a la protección de estas especies amenazadas.

\section{Conclusiones}

1) Queda remarcada la importancia de aumentar el nivel de conocimiento de las especies amenazadas para la elaboración adecuada de planes y actuaciones de conservación. Los criterios del grado de amenaza son más fidedignos cuanto más exacto sea este conocimiento, haciendo constar la relevancia de los datos corológicos y demográficos que permiten la estimación más realista de las proyecciones poblacionales.

2) Uno de los riesgos más impactantes sobre los taxones amenazados es la competencia con otras especies alóctonas e invasoras. Por eso sería adecuado mantener un seguimiento de estas especies para valorar el impacto que suponen sobre el ecosistema que invaden.

Es digno de mencionar el aumento en el porcentaje del número de citas de amenaza por especies alóctonas, en comparación con la distribución en el Libro Rojo, donde las amenazas registradas eran, en su mayoría, debido a la alteración del hábitat. Esto da un perfil completamente distinto, pero no por ello es el prevalente, dado que las amenazas citadas a lo largo de este trabajo son sumatorias a las ya conocidas en el Libro Rojo.

3) La otra gran amenaza es la actividad humana y los efectos indirectos que de ella se derivan, como son el cambio climático y la contaminación del medioambiente. El ser humano debe ser consciente de que sus acciones afectan a este. La conservación de las especies amenazadas no se ciñe exclusivamente a la implementación de medidas de gestión y planes de actuación, sino también a la concienciación social.

4) Asimismo, la implementación de técnicas genéticas para la identificación de las especies permite evitar los errores de la identificación puramente morfológica. La correcta identificación es esencial para no caer en juicios erróneos en el momento de evaluar las poblaciones y los peligros, que se traducen inmediatamente en la asignación de criterios del grado de amenaza.

5) Una concepción muy extendida es el hecho de que los endemismos son potencialmente más vulnerables por su limitación territorial, pero no es así. Las especies no endémicas son más vulnerables, por el hecho de que nuestro territorio puede suponer el límite de distribución poblacional y, al estar en el margen, no gozan de la posibilidad de rescate tanto genético como poblacional del grueso de la población.

6) La ausencia de planes de recuperación y conservación de las especies amenazadas, de obligada elaboración por la normativa vigente, ha puesto la situación de algunas especies en una posición crítica. Por este motivo, algunos parques naturales se han visto obligados a elaborar sus propios programas de conservación (SEFA o plan de conservación) para poder salvaguardar algunas especies en riesgo, mientras se espera a que los planes pertinentes sean aprobados. 


\section{Bibliografía}

Anton, M., Artola, J., Cristóbal, J., Guinart, D., Gómez, J., Herrando, S. (2014). Pla de conservació del Parc Natural del Montseny. Reserva de la Biosfera. Barcelona: Diputació de Barcelona.

Aparicio, D., Katenhusen, O., Gustamante, L., Clavell, J., Aymerich, P. (2013). «Reaparició d'una espècie extingida a Catalunya: Spirodela polyrrhiza (L.) Schleid. al pantà de Vallvidrera (serra de Collserola, Barcelona)». Butll. Inst. Catalana Hist. Nat., 77, pp. 161-163.

Aymerich, P. (2012). «Potamogeton i Zannichellia a la conca mitjana del riu Llobregat (Catalunya, NE península Ibèrica)». Orsis, 26, pp. 57-89.

Aymerich, P. (2013a). «Notes sobre algunes plantes rares o amenaçades als Pirineus catalans». Butll. Inst. Catalana Hist. Nat., 77, pp. 5-26.

Aymerich, P. (2013b). «Sobre algunes espècies al-lòctones a l'alt Segre (NE de la península Ibèrica), noves o molt rares per a la flora catalana». Orsis, 27, pp. 195-207.

Aymerich, P. (2015). «Notes florístiques de les conques altes dels rius Segre i Llobregat. III». Orsis, 29, pp. 91-118.

Aymerich, P. (2016). «Contribució al coneixement de la flora al-lòctona del nord i el centre de Catalunya». Orsis, 30, pp. 11-40.

Aymerich, P. (2017). «Notes sobre la flora del Pallars i l'alt Aran». Orsis, 31, pp. 99-114.

Aymerich, P., Tenas, B., Vigo, J. (2010). «Notes florístiques del Ripollès (Pirineus orientals)». Acta Botanica Barcinonensia, 53, pp. 11-26.

BıoC. (2012). «L'esperó de Bolòs, retrobat a St. Llorenç». Portal de Biologia de la Conservació de Plantes. Disponible en: http://bioc.org.es/bioc/index.php?option=com_content\&view=article\&id=59 1\%3Alespero-de-bolos-retrobat-a-st-llorenc\&catid=1\%3Alatest\&Itemid=53 [acceso: 13 marzo 2020].

BioC. (2020). Portal de Biologia de la Conservació de Plantes. Disponible en: http://biologia-conservacio. org/bioc/ [acceso: 12.03.2020].

BlAnchÉ, C. (2013). «Raresa botànica. Plantes rares i amenaçades. Prioritats per a la conservació de la biodiversitat en un món canviant. Una visió des de Catalunya». Discurs d'ingrés a la Reial Acadèmia de Farmàcia de Catalunya. Barcelona.

BOE (2007). Ley 42/2007, de 13 de diciembre, del Patrimonio Natural y de la Biodiversidad. Boletín Oficial del Estado (14.12.2007).

BOE (2011). Real Decreto 139/2011, de 4 de febrero, para el desarrollo del Listado de Especies Silvestres en Régimen de Protección Especial y del Catálogo Español de Especies Amenazadas. Boletín Oficial del Estado (23.02.2011).

BOE (2015a). Ley 33/2015, de 21 de septiembre, por la que se modifica la Ley 42/2007, de 13 de diciembre, del Patrimonio Natural y de la Biodiversidad. Boletín Oficial del Estado (22.09.2015).

BOE (2015b). Orden AAA/1771/2015, de 31 de agosto, por la que se modifica el anexo del Real Decreto 139/2011, de 4 de febrero, para el desarrollo del Listado de Especies Silvestres en Régimen de Protección Especial y del Catálogo Español de Especies Amenazadas. Boletín Oficial del Estado (03.09.2015).

Bosch Daniel, M., Mampel Marimon, A., Bachis i Prim, I., Massó i Alemán, S., López-Pujol, J., BlanchÉ VERGÉs, C. (2019a). «Pol-linització i reproducció de l'esperó de Bolòs (Delphinium bolosii) al Parc Natural de Sant Llorenç del Munt i l'Obac». IX Trobada d'Estudiosos de Sant Llorenç del Munt i l'Obac, Barcelona: Diputació de Barcelona, pp. 359-377.

Carrera, D., Serrano, A., Vila, M., Dalmases, C. (2016). «Avaluació de l'estat de la flora vascular amenaçada a la Xarxa de Parcs Naturals de la Diputació de Barcelona. El Programa SEFA». Llibre de resums de les IV Jornades de Conservació de Flora i Funga, Olot: Fundació d'Estudis Superiors d'Olot, p. 12.

Conesa, J. A., Galán de Mera, A., Pedrol, J. (2016). «Noves lemnàcies al riu Segre». Butll. Inst. Catalana Hist. Nat., 80, pp. 59-60.

CREAF (2020). CREAF. Disponible en: http://www.creaf.cat/es [acceso: 27 abril 2020].

Curto, R., Arrufat, M., Beltran, J., CreiX, À., Fontanet, J., Royo, F. (2013). «Retrobada a Catalunya (NE de la península Ibèrica) una població de Spirodela polyrhiza (Araceae)». Orsis, 27, pp. 141-150. 
Diputació de BARcelona (2020). «Qué es la Red». Xarxa de Parcs Naturals. Disponible en: https://parcs. diba.cat/es/que-es-la-xarxa [acceso: 11 mayo 2020].

DOGC (2008). DECRET 172/2008, de 26 d'agost, de creació del Catàleg de flora amenaçada de Catalunya. Diari Oficial de la Generalitat de Catalunya (28.08.2008).

DOGC (2015) RESOLUCIÓ AAM/732/2015, de 9 d'abril, per la qual s'aprova la catalogació, descatalogació i canvi de categoria d'espècies i subespècies del Catàleg de flora amenaçada de Catalunya. Diari Oficial de la Generalitat de Catalunya (20.04.2015).

Generalitat de Catalunya (2020a) «Estratègia Catalana de Conservació de la Flora i plans de recuperació i conservació». Disponible en: http://mediambient.gencat.cat/ca/05_ambits_dactuacio/patrimoni_ natural/flora-autoctona-protegida/estrategies/ [acceso: 11 mayo 2020].

Generalitat DE Catalunya (2020b). «Estrategia catalana de conservación ex situ». Disponible en: http:// mediambient.gencat.cat/es/05_ambits_dactuacio/patrimoni_natural/flora-autoctona-protegida/ estrategies/estrategia_catalana_conservacio_ex_situ/ [acceso: 11 mayo 2020].

GNO. (2020a) Grup de Naturalistes d'Osona. Disponible en: http://www.gno.cat/ [acceso: 27 abril 2020].

GuARDiola, M., GutiÉRREz, C. (2012) «Redescoberta d'Isoetes durieui Bory a la Serralada Litoral Central catalana». II Monografies dels parcs de la Serralada Litoral Central i VI Monografies del Montnegre i el Corredor. Diputació de Barcelona, pp. 293-299.

Guardiola, M., Petit, A., Carrillo, E., Pérez-HaAse, A., Batriu, E., Ninot, J. M., Sáez, L. (2013b). «Aportacions a la flora dels Pirineus central (II)». Orsis, 27, pp. 261-286.

GuARdiola, M., Romera, X. (2018). «Reaparició de Reseda hookeri (Resedaceae) a les platges del Maresme, una espècie considerada extingida a Catalunya». Butll. Inst. Catalana Hist. Nat., 82, pp. 69-71.

Gutiérrez, C., GuARDiola, M. (2012). «Creació d'una base de dades de flora vascular del Parc del Garraf i proposta de llista vermella provisional». VI Monografies del Garraf i d'Olèrdola. Diputació de Barcelona, pp. 106-112.

IbARs, A. M., Estrelles, E. (2011). «Caracterización de la germinación de las esporas y desarrollo de los gametofitos de Asplenium majoricum». Programa y Libro de resúmenes del V Congreso de Biología de la Conservación de Plantas. Es Mercadal (Menorca): Universitat de les Illes Balears y Sociedad Española de Biología de la Conservación de Plantas, p. 154.

IUCN. (2020) UICN, Unión Internacional para la Conservación de la Naturaleza. Disponible en: https:// www.iucn.org/es [acceso: 16 marzo 2020].

Jeschke, J. M., Bacher, S., Blackburn, T. M., Dick, J. T. A., Essl, F., Evans, T. et al. (2014) «Defining the impact of non-native species». Conserv. Biol., 28, pp. 1188-1194.

Jover Benjumea, M., Saura-Mas, S., Benejam Vidal, L. (2014) «Hydrocotyle vulgaris una espècie amenaçada retrobada al Baix Empordà». Butll. Inst. Catalana Hist. Nat., 78, pp. 81-82.

López-Alvarado, J., Сobacho, I., Arán, V. J., Rosato, M., Rosselló, J. A., Sáez, L. (2017) «Limonium ilergabonum (Plumbaginaceae), a new species from Northeastern Iberian peninsula». Phytotaxa, 331, pp. 199-212.

López-Pujol, J., Martinell, M. C., Massó, S., Blanché, C., Molero, J. (2010) «Seseli farrenyi: un endemisme empordanès en vies d'extinció?» Collect. Bot., 29, pp. 51-58.

Martinell, M. C., López-Pujol, J., Blanché, C., Molero, J., Sàez, L. (2011a) «Conservation assessment of Aquilegia paui (Ranunculaceae): A case study of an extremely narrow endemic». Oryx, 45, pp. 187190.

Martinell, M. C., Rovira, A., Blanché, C., Bosch, M. (2011b) «Shift towards autogamy in the extremely narrow endemic Aquilegia paui and comparison with its widespread close relative A. vulgaris (Ranunculaceae)». Plant Syst. Evol., 295, pp. 73-82.

Ministerio PARA la Transición ECológica y El Reto Demográfico (2020b) «Conservación ex situ». Disponible en: https://www.miteco.gob.es/es/biodiversidad/temas/conservacion-de-especies/ conservacion-ex-situ/default.aspx [acceso: 11 mayo 2020].

Oliver Martínez-Fornés, X. (2012) «Poblacions de Woodsia alpina (Bolton) S.F. Gray a la comarca del Ripollès». Llibre de resums de les III Jornades de Conservació de Flora. Lleida: Edicions de la Universitat de Lleida, p. 35.

OLIVER, X. (2018) «Revaluació i actuacions clau en conservació de plantes vasculars de la Garrotxa a partir 
Edusfarm 13 (2021), 17-33

ISSN: $1886-6271$

dels criteris de la Llista Vermella (IUCN , 2017)». Annals de la Delagació de la Garrotxa de la Inst. Cat. Hist. Nat., 8, pp. 193-217.

Oliver, X., BÉJAR, X., Lockwood, M., Drake, I., VILAsís, D., Cos, I. et al. (2010) «Noves dades de flora vascular de la Garrotxa, 2009». Annals de la Delegació de la Garrotxa de la Inst. Cat. Hist. Nat., 4, pp. 71-77.

Oliver, X., Villegas, N., Zapata, J., RABASA, M., Vilasís, D., Béjar, X. (2016) «Novetats i aportacions fetes durant l'any 2013 als catàlegs de flora de la Garrotxa». Annals de la Delagació de la Garrotxa de la Inst. Cat. Hist. Nat., 8, pp. 165-178.

RINCoN, P. (2020) «Allergy impact from invasive weed 'underestimated'» $B B C$. Disponible en: https:// www.bbc.com/news/science-environment-52367688 [acceso: 26 mayo 2020].

SÁEz, L., AYMERICH, P., BlAnChÉ, C. (2010) Llibre Vermell de les plantes vasculars endèmiques i amenaçades de Catalunya. Barcelona: Argania editio.

Soriano, I., Aymerich, P. (2017) «Precisions sobre la població i l'estatus de Carex brevicollis (Cyperaceae) a Catalunya». Butll. Inst. Catalana Hist. Nat., 81, pp. 117-119.

UICN. (2012) Categorías y Criterios de la Lista Roja de la UICN: Version 3.1. Segunda edición. Gland, Suiza y Cambridge, Reino Unido, 2001. 